\title{
Silver Nanowire based Flexible, Transparent, Wideband Antenna for 5G Band Application
}

\author{
Weiwei Li, Azat Meredov, and Atif Shamim \\ Division of Computer, Electrical, and Mathematical Sciences and Engineering \\ King Abdullah University of Science and Technology (KAUST) \\ Thuwal 23955-6900, Saudi Arabia \\ weiwei.li@kaust.edu.sa, azat.meredov@kaust.edu.sa, atif.shamim@kaust.edu.sa
}

\begin{abstract}
A transparent, flexible antenna can be utilized on car windows, solar cells, and similar applications to make use of the existing space for communication. In this work, a flexible, transparent, and wideband mm-wave monopole antenna has been designed, fabricated and tested. The proposed antennas have been realized by inkjet printing of custom made silver nanowire (Ag NW) ink. The antenna shows ultra-wide bandwidth up to $26 \mathrm{GHz}$ (from $18 \mathrm{GHz}$ to $44 \mathrm{GHz}$ ), high radiation efficiency of $55 \%$ and a maximum gain of $1.45 \mathrm{dBi}$. In addition, more than $90 \%$ transparency has been obtained due to the high transparency of the Ag NW and the slot structure. The excellent performance enables the proposed antenna suitable for various applications.
\end{abstract} wave

Keywords—-transparent antenna; silver nanowire; flexible; $\mathbf{m m -}$

\section{INTRODUCTION}

The Internet of Things (IoT) based on wireless communication has become an inherent part of daily life. As the major part of the wireless communication, antennas are gaining more and more attention, especially the optical transparent antennas, which eliminating the visual impact and represents an attractive scheme in various applications of solar panel, satellite, and smart vehicle. $5 \mathrm{G}$ is one future application allowing faster data transfer by utilizing wider frequency bandwidth [1]. Previous works on triangular monopole have shown wide frequency bandwidth [2], making it suitable for $5 \mathrm{G}$ applications.

Generally, the conventional antennas showing optical transparency are made of transparent conducting oxides, for example, Indium Tin Oxide (ITO), which also shows high conductivity [3, 4]. However, the extensively studied ITO suffers from many drawbacks, such as high cost due to the rare material Indium, complex fabrication procedures and limited mechanical flexibility due to brittle behavior. Except for the conductive materials, the substrates widely used in the transparent antennas, are brittle [5], which also limit the potential applications in flexible and wearable devices.

In this work, a wideband monopole antenna with more than $90 \%$ transparency has been designed and fabricated with the help of inkjet printing technique. The proposed antenna is also flexible and bendable due to the flexible nature of the substrate and the silver nanowire (Ag NW) prepared in the lab.

\section{ANTENNA DESIGN AND FABRICATION}

The antenna designs are carried out using a 3D full-wave simulator CST Microwave Studio 2018. Flexible and low loss dielectric substrate with a permittivity of $\varepsilon=2.6$ and a $\tan \delta=0.0044$ at $24 \mathrm{GHz}$ is selected as the substrate. The thickness of the substrate is $0.05 \mathrm{~mm}$. The conductivity of the antenna is $10^{5} \mathrm{~S} / \mathrm{m}$.

\section{A. Antenna design}

The proposed transparent antenna in this paper is a triangular monopole antenna with a microstrip line feeding. To obtain a wideband frequency range, a $60^{\circ}$ angle has been chosen for the triangular monopole. The length of the antenna has been optimized to get center frequency around $30 \mathrm{GHz}$. The detailed geometry parameters and $S$-parameters of the transparent antenna has been shown in Fig. 1. The optimized transparent antenna shows a bandwidth of $26 \mathrm{GHz}$, radiation efficiency of $\sim 62 \%$ and maximum gain of $1.5 \mathrm{dBi}$. The transparency of the antenna has been further increased by introducing slots in the antenna and ground plane. Due to the small size of the antenna, it is hard to connect SMA to this antenna. Thus, CPWG feed has been added to the antenna to make probing feasible.

(a)
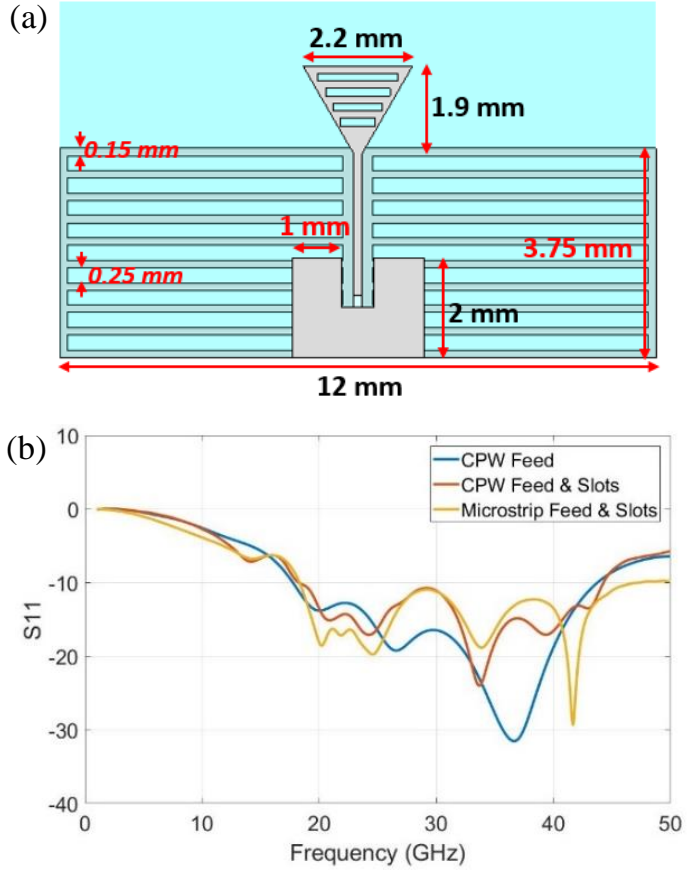

Fig. 1. (a) Structure of the triangle monopole antenna. (b) S-parameters of the transparent slotted and plane triangular monopole antennas with $\mathrm{CPW}$ and microstrip line feeds. 
For comparison, the same antenna has been studied with a silver nanoparticle (Ag NP) ink which has higher conductivity $\left(10^{7} \mathrm{~S} / \mathrm{m}\right)$, but much lower transparency. Attributing to the high conductivity, the Ag NP antennas show higher efficiency (more than 90\%) and larger maximum gain (more than $3 \mathrm{dBi}$ ) comparing to the Ag NW antennas (see Table I).

\section{B. Antenna fabrication}

An inkjet printing technique has been used to fabricate the antennas. For the non-transparent antennas, Ag NP ink was directly deposited onto the substrate to form the desired monopole antennas and ground layers. For the Ag NW antennas, a polymer ink was printed onto the substrate with desired patterns. Then, Ag NW ink was deposited through spin-coating. Finally, the fabrication process was completed by immersing the sample into an organic solvent to remove the polymer layer. Fig. 2 shows the fabricated antennas and Ag NW antenna under different bending conditions, demonstrating the flexibility.
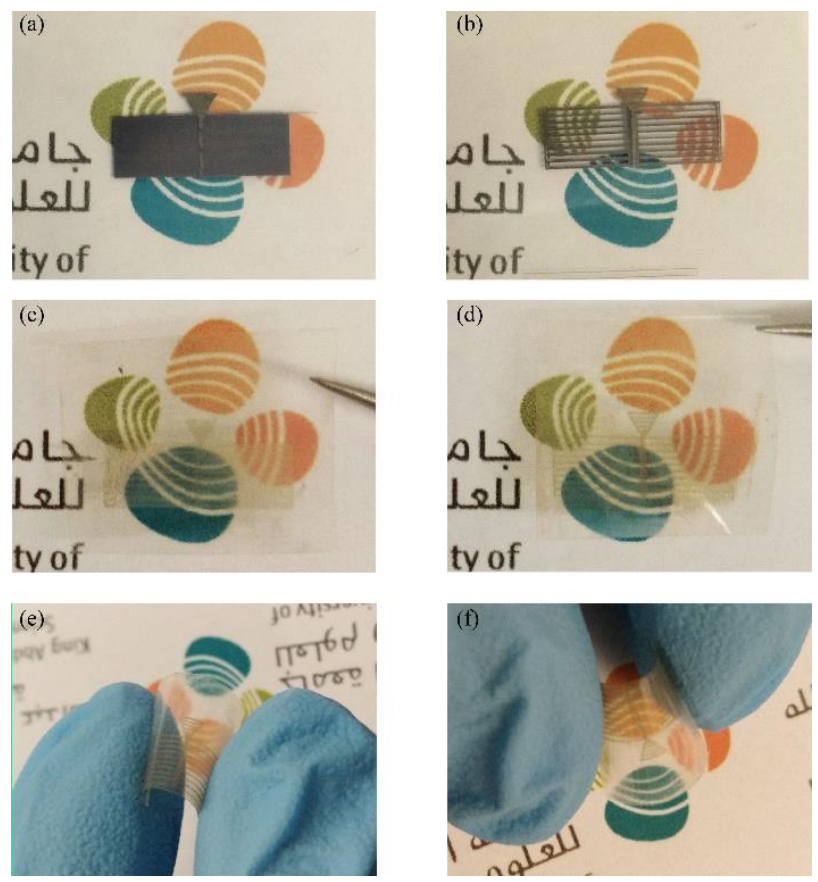

Fig. 2. (a) Antenna with Ag NP ink, (b) antenna with Ag NP ink and slots, (c) antenna with $\mathrm{Ag} \mathrm{NW}$ ink, and (d) antenna with $\mathrm{Ag} \mathrm{NW}$ ink and slots. (e) The $\mathrm{Ag}$ NW antenna under bending convex condition. (f) The Ag NW antenna under bending concave condition.

\section{ANTENNA TRANSPARENCY CHARACTERIZATION}

For the Ag NP-based antennas, the transmittance is equal to the area of the Ag divided by the total area [6]. For the Ag NW antennas, the transmittance has been measured in the visible light wavelength (Fig. 3). The results are compared and shown in Table I. It is reasonable to find that the Ag NW antenna with slots both in monopole antenna and ground layer shows the best transparency, which could be as high as $91.2 \%$.

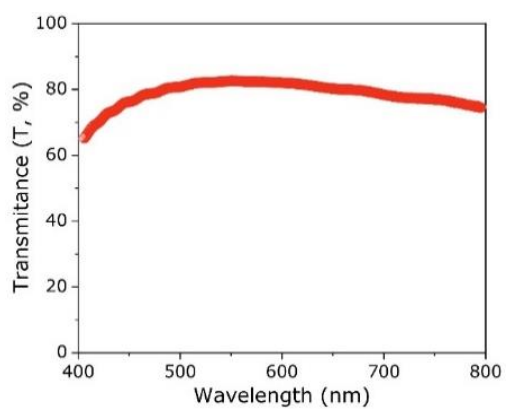

Fig. 3. UV-Vis spectra of the Ag NW antenna without slot structure.

\section{TABLE I. COMPARISON FOR THE FABRICATED ANTENNAS}

\begin{tabular}{|c|c|c|c|c|}
\hline Antenna type & $\begin{array}{c}\text { Bandwidth } \\
(\mathbf{G H z})^{\boldsymbol{a}}\end{array}$ & $\begin{array}{c}\text { Efficiency } \\
(\boldsymbol{\%})^{\boldsymbol{a}}\end{array}$ & $\begin{array}{c}\text { Max Gain } \\
(\mathbf{d B i})^{\boldsymbol{a}}\end{array}$ & $\begin{array}{c}\text { Transmittance } \\
(\%)\end{array}$ \\
\hline Ag NP & 17 & 92 & 3.4 & 0 \\
\hline $\begin{array}{c}\text { Ag NP with } \\
\text { slot }\end{array}$ & 6.8 & 90 & 3.2 & 42.3 \\
\hline Ag NW & 26 & 62 & 1.5 & 82.4 \\
\hline $\begin{array}{c}\text { Ag NW with } \\
\text { slot }\end{array}$ & 26 & 55 & 1.45 & 91.2 \\
\hline \multicolumn{5}{|c|}{ a. Simulated results. The measured results will be provided. }
\end{tabular}

\section{CONCLUSION}

In this work, a wideband, flexible, and transparent triangular monopole antenna has been designed for $5 \mathrm{G}$ applications. The Ag NW ink has been prepared to be used in the fabrication of the transparent antennas with the help of inkjet printing technique. The Ag NW based antenna shows ultra-wide bandwidth up to $26 \mathrm{GHz}$, high radiation efficiency of $55 \%$ and a maximum gain of $1.45 \mathrm{dBi}$. Notably, more than $90 \%$ transparency has been obtained for the Ag NW antenna.

\section{REFERENCES}

[1] N. O. Parchin, M. Shen, and G. F. Pedersen, (2016, October). End-fire phased array $5 \mathrm{G}$ antenna design using leaf-shaped bow-tie elements for 28/38 GHz MIMO applications. In Ubiquitous Wireless Broadband (ICUWB), 2016 IEEE International Conference on (pp. 1-4). IEEE.

[2] C.C. Lin, Y.C. Kan, L.C. Kuo, and H.R. Chuang, (2005). A planar triangular monopole antenna for UWB communication. IEEE Microwave and wireless components letters, 15(10), pp.624-626.

[3] C. Kocia, and S. V. Hum, (2016). Design of an optically transparent reflectarray for solar applications using indium tin oxide. IEEE Trans. Antennas Propag., 64(7), 2884-2893.

[4] N. I M. Ali, N Misran, M F Mansor and M F Jamlos, (2017, May). Transparent solar antenna of $28 \mathrm{GHz}$ using transparent conductive oxides (TCO) thin film. In Journal of Physics.: Conf. Series vol. 852, No. 1, p. 012036. IOP Publishing.

[5] G. Sun, B. Muneer and Q. Zhu, (2014, July). A study of microstrip antenna made of transparent ITO films. In 2014 IEEE Antennas and Propagation Society International Symposium (APSURSI) (pp. 18671868). IEEE.

[6] K. Neytsa, A. Real, M. Marescaux, S. Mladenovski, and J. Beeckman, (2008). Conductor grid optimization for luminance loss reduction in organic light emitting diodes. J. of App. Phys., 103(9), 093113. 